\title{
Effect of upper gastrointestinal bleeding on prognosis of middle- aged patients with acute ischemic stroke: a retrospective study
}

\author{
Tinggang Wang ${ }^{1}$, Dongxiao $\mathrm{Zhu}^{2}$, Liang Kong ${ }^{1}$, Chenyan $\mathrm{Mu}^{1}$, Chaosheng $\mathrm{Li}^{1}$, Lingling $\mathrm{Hu}^{1}$ \\ ${ }^{1}$ Department of Neurology, Affiliated Hospital of Jiangnan University, Wuxi, China; ${ }^{2}$ Department of Ultrasonography, Affiliated Hospital of Jiangnan \\ University, Wuxi, China \\ Contributions: (I) Conception and design: T Wang, L Hu; (II) Administrative support: L Hu; (III) Provision of study materials or patients: All authors; \\ (IV) Collection and assembly of data: T Wang, D Zhu, L Kong, C Mu, C Li; (V) Data analysis and interpretation: T Wang; (VI) Manuscript writing: \\ All authors; (VII) Final approval of manuscript: All authors. \\ Correspondence to: Lingling Hu. Department of Neurology, Affiliated Hospital of Jiangnan University, Hefeng Road, Wuxi 214000 , China. \\ Email: 317195209@qq.com.
}

Background: Upper gastrointestinal bleeding (UGIB) is a common complication of acute ischemic stroke (AIS), but the effect of UGIB on the prognosis of middle-aged AIS patients is not clear.

Methods: Patients with AIS admitted to our hospital from January 2011 to December 2015 were eligible to be included in this study. All included patients were divided into UGIB and non-UGIB groups. Some clinical characteristics were retrospectively collected. Primary outcomes were all-cause mortality within 1, 3, and 5 years, as well as the incidence of stroke recurrence. Cox proportional hazards regression analyses were used to determine the effect of UGIB on 5-year mortality and the incidence of stroke recurrence. Logistic regression was also used to identify the predictors of UGIB in AIS patients.

Results: A total of 405 AIS patients were included in this study and then divided into UGIB and nonUGIB groups. The mean age of the UGIB group and non-UGIB group was $61.5 \pm 9.6$ and $53.1 \pm 14.0$ years, respectively $(\mathrm{P}<0.001)$. The baseline score of the National Institute of Health Stroke Scale (NIHSS) was significantly higher in the UGIB group than in the non-UGIB group $(\mathrm{P}<0.001)$. AIS patients in the UGIB group had a higher 1-, 3-, and 5-year mortality and a higher incidence of stroke recurrence (all $\mathrm{P}<0.001$ ). Kaplan-Meier curves showed that AIS patients with UGIB had a higher 5-year mortality and a higher incidence of stroke recurrence (both $\mathrm{P}<0.001$ ). Cox proportional hazards regression models indicated that the occurrence of UGIB, older age, a high NIHSS score, and stroke recurrence were related to a higher 5-year mortality. Similarly, the occurrence of UGIB, older age, a high NIHSS score, and hypertension increased the incidence of stroke recurrence. According to the multivariate logistic regression analysis, older age, a high NIHSS score, and previous anticoagulant use were identified as predictors of UGIB.

Conclusions: UGIB has important effects on the prognosis of AIS patients. The incidence of UGIB increases with older age, a high NIHSS score, and previous anticoagulant use, which provides important evidence for the treatment and nursing of AIS patients.

Keywords: Upper gastrointestinal bleeding (UGIB); acute ischemic stroke (AIS); middle-age; mortality; recurrence

Submitted Mar 24, 2021. Accepted for publication May 06, 2021.

doi: 10.21037/apm-21-907

View this article at: http://dx.doi.org/10.21037/apm-21-907 


\section{Introduction}

Acute ischemic stroke (AIS) is a common cerebrovascular disease throughout the world and accounts for two-thirds of all stroke patients (1). More recently, stroke patients are tending to be younger and often have a poor prognosis (2). Over the course of treatment, patients can also develop other complications that adversely affect their prognosis. Upper gastrointestinal bleeding (UGIB) is a common complication of AIS, with an incidence rate of $1.2-8.1 \%$ (3-6). Many risk factors are related to the incidence of UGIB, including age, gender, hypertension, history of peptic ulcer, previous UGIB, and stroke subtype (7-9). According to previous studies, UGIB is related to a worse prognosis in AIS patients $(10,11)$. In their retrospective study, Chou et al. reported that the occurrence of UGIB increased the 3-year mortality rate of acute stroke patients (10). Du et al. also found that UGIB increased the incidence of stroke recurrence after AIS (11).

Most previous studies on the effects of UGIB have focused on the prognosis of elderly patients with AIS. Age has been verified as an important independent risk factor for UGIB, and elderly patients with AIS are more likely to suffer from UGIB than younger patients $(7,8)$. However, the proportion of middle-aged stroke patients has increased in recent years. Although the prognosis for middle-aged stroke patients is better than that of elderly patients (12), the increasing number of middle-aged patients with stroke makes the study of their prognosis an important focus of research. Considering the association between UGIB and prognosis in elderly patients with AIS, it is important to determine whether this relationship is similar in middle-aged stroke patients, but to date this has not been investigated.

This was a retrospective study enrolling AIS patients from a single center to determine mortality and stroke recurrence in middle-aged patients. In addition, the incidence of UGIB was recorded and the effect of UGIB on the prognosis of AIS was determined using a multivariate Cox regression analysis. We present the following article in accordance with the STROBE reporting checklist (available at http://dx.doi.org/10.21037/apm-21-907).

\section{Methods}

\section{Study participants}

Patients with AIS who were admitted to our hospital from January 2011 to December 2015 and who met the inclusion and exclusion criteria were included in this study. The diagnosis of AIS was defined according to specific symptoms such as limb weakness, unconsciousness, and syncope, and then confirmed by brain imaging using computed tomography or magnetic resonance. Only middle-aged AIS patients who were 40-65 years old were included in this study. The following patients were excluded: (I) patients with a previous history of AIS; (II) patients who were sent to our hospital after more than 3 days; (III) patients who were transferred to other hospitals or returned home without proper treatment during hospitalization. All procedures performed in this study involving human participants were in accordance with the Declaration of Helsinki (as revised in 2013). The study was approved by institutional ethics board of Affiliated Hospital of Jiangnan University (No.: 02019050). Written informed consent from patients was not required due to the retrospective nature of the study.

\section{Study data}

All included patients were divided into UGIB and nonUGIB groups. UGIB was defined according to specific symptoms such as hematemesis and melena, and then confirmed by endoscopy. The following data were collected by the investigators: age; gender; baseline score of the National Institute of Health Stroke Scale (NIHSS); stroke subtype [including partial anterior circulation infarct (PACI), total anterior circulation infarct (TACI), lacunar infarction (LACI), and posterior circulation infarct (POCI), confirmed by computed tomography or magnetic resonance]; comorbidities (including hypertension, diabetes mellitus, hyperlipidemia, anemia, coronary artery disease, and liver cirrhosis); history of smoking; history of alcoholism; previous anticoagulant use; previous antiplatelet use; and previous non-steroidal anti-inflammatory drug (NSAID) use. These data were collected by several investigators and summarized by one investigator for screening and sorting.

\section{Study outcomes}

All patients were followed up for a maximum of 5 years. The primary outcomes in this study were all-cause mortality within 1,3 , and 5 years, as well as the incidence of stroke recurrence. Stroke recurrence was defined as any AIS event occurring during the follow-up period. The secondary outcomes included length of hospital stay and in-hospital mortality. 


\section{Statistical analysis}

Continuous variables such as age, baseline NIHSS score, and length of hospital stay were reported and expressed as means and standard deviations. Categorical variables such as gender, subtype of stroke, comorbidities, history of smoking and alcoholism, previous drug use, mortality and stroke recurrence were reported and expressed as numbers and percentages. Kaplan-Meier curves and the log-rank test were performed to compare the 1-, 3-, and 5-year mortality rates of the UGIB and non-UGIB groups. Univariate and multivariate Cox proportional hazards models were used to determine the effect of UGIB on 5-year mortality and the incidence of stroke recurrence. In addition, logistic regression was used to identify the predictors of UGIB in AIS patients. All statistical analyses were performed using SPSS 18.0 (IBM Inc. NY, USA) and a P value $<0.05$ was considered statistically significant.

\section{Results}

According to the inclusion and exclusion criteria, a total of 405 AIS patients were included in this study. Their clinical characteristics are listed in Table 1. Because this study only included middle-aged AIS patients, the mean age of included patients was $54.1 \pm 13.8$ years old. Male patients accounted for $58.5 \%$ of the total sample, and the mean NIHSS baseline score for all patients was $4.8 \pm 2.7$ points. PACI was the most common subtype of AIS. In regard to comorbidities, 243 patients (60.0\%) had hypertension, 98 patients $(24.2 \%)$ had diabetes mellitus, 29 patients $(7.2 \%)$ had hyperlipidemia, 21 patients (5.2\%) had anemia, and 25 patients $(6.2 \%)$ had coronary artery disease. In addition, two patients $(0.5 \%)$ were diagnosed with liver cirrhosis. There were 154 patients $(38.0 \%)$ with a history of smoking and 38 patients $(9.4 \%)$ with a history of alcoholism. The data on medication use were as follows: 43 patients $(10.6 \%)$ had previously used anticoagulant drugs, 166 patients (41.0\%) had previously used antiplatelet drugs, and 57 patients (14.1\%) had previously used NSAIDs.

The primary and secondary outcomes are listed in Table 2. For all included patients, 1-year mortality was 5.4\% (22 patients), 3-year mortality was $9.6 \%$ (39 patients), and 5 -year mortality was $15.6 \%$ (63 patients). The incidence of stroke recurrence was $7.7 \%$ (31 patients). In total, 14 patients died in hospital and the mean length of hospital stay was $6.8 \pm 3.2$ days.

Included patients were then divided into UGIB and
non-UGIB groups. Their clinical characteristics are listed in Table 1. Results showed that the mean age of the UGIB group was $61.5 \pm 9.6$ years compared with $53.1 \pm 14.0$ years in the non-UGIB group, and this difference was statistically significant $(\mathrm{P}<0.001)$. There were more male patients in the UGIB group (70.8\%) than in the non-UGIB group (56.9\%), however, this difference was not significant $(\mathrm{P}=0.065)$. The baseline NIHSS score was significantly higher in the UGIB group $(11.3 \pm 2.3$ points) than in the non-UGIB group (4.0 \pm 1.2 points) $(\mathrm{P}<0.001)$. Moreover, stroke subtypes were different between the two groups. There were more PACI patients in the UGIB group $(\mathrm{P}=0.041)$ and more TACI patients in the non-UGIB group $(\mathrm{P}=0.016)$. There were more patients with a prior history of anticoagulant drug use in the UGIB group (22.9\%) than in the non-UGIB group $(9.0 \%)$, and this difference was statistically significant $(\mathrm{P}=0.003)$. In regard to outcomes, AIS patients in the UGIB group had a higher 1-, 3-, and 5-year mortality rate, a higher incidence of stroke recurrence, a higher in-hospital mortality rate, and a longer length of hospital stay (all $\mathrm{P}<0.001)$ (Table 2).

The Kaplan-Meier curves of 5-year mortality and incidence of stroke recurrence are shown in Figures 1 and 2. Both curves show that AIS patients with UGIB had a higher 5 -year mortality and a higher incidence of stroke recurrence than AIS patients without UGIB (both $\mathrm{P}<0.001$ according to the log-rank test).

Results of the Cox proportional hazards analyses of the risk factors for 5-year mortality are shown in Table 3. Multivariate Cox regression indicated that the occurrence of UGIB, older age, a high NIHSS score, and stroke recurrence were related to a higher 5-year mortality rate. Univariate Cox regression indicated that hypertension may affect 5 -year mortality. The risk factors for stroke recurrence are also shown in Table 4. Similar to the risk factors for 5-year mortality, the occurrence of UGIB, older age, a high NIHSS score, and hypertension were associated with an increase in the incidence of stroke recurrence according to the univariate Cox regression analysis.

Finally, predictors of UGIB in AIS patients are shown in Table 5. Older age, a high NIHSS score, and previous anticoagulant use were identified as predictors of UGIB in AIS patients according to the multivariate logistic regression analysis.

\section{Discussion}

Previous studies have tended to focus on research 
Table 1 Clinical characteristics of total AIS patients, and AIS patients with and without UGIB

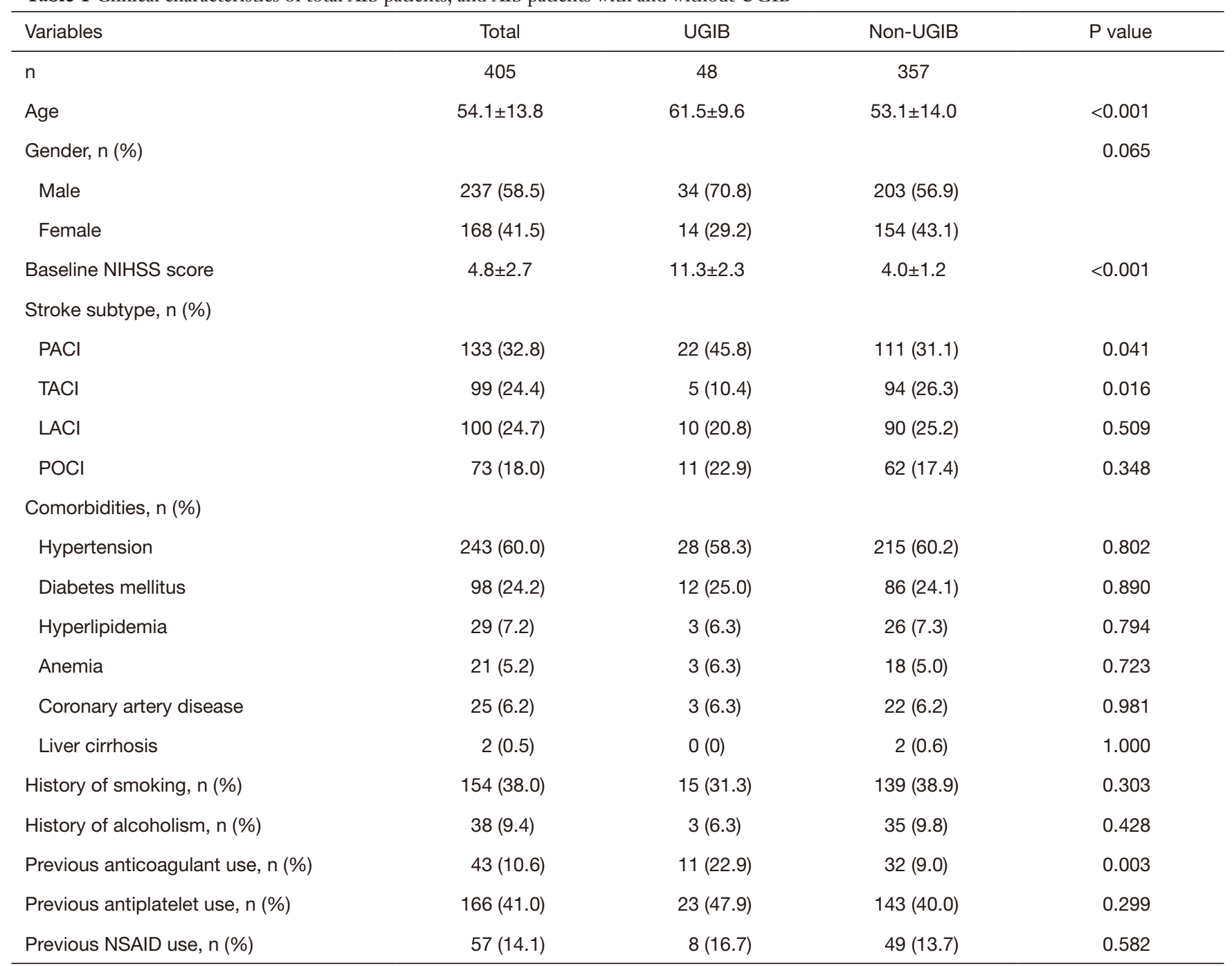

AIS, acute ischemic stroke; UGIB, upper gastrointestinal bleeding; NIHSS, National Institute of Health Stroke Scale; PACI, partial anterior circulation infarct; TACI, total anterior circulation infarct; LACI, lacunar infarction; POCI, posterior circulation infarct; NSAID, non-steroidal anti-inflammatory drug.

concerning elderly AIS patients. However, research of middle-aged AIS patients is becoming more important considering the increased incidence of AIS in this category of patient. We included AIS patients aged from 40-65 years old, and the mean age of patients in our study was $54.1 \pm$ 13.8 years old. To our knowledge, this is also the first study investigating the relationship between UGIB and prognosis in AIS patients.

UGIB was verified as a significant risk factor for 5-year mortality in our study, which was similar to findings from previous studies of elderly patients $(10,13)$. UGIB itself poses harm to patients, and can result in a mortality rate of up to $16-24 \%$ (14-16). The harm to AIS patients is further magnified due to the poor condition of the patients themselves. Also, age has been verified as an independent risk factor for mortality in elderly AIS patients (17-19). In our study, older age remained an important risk factor for mortality in relatively younger AIS patients. NIHSS is a comprehensive scale of consciousness, limb movement, ataxia, balance and other aspects and represents the severity of stroke. The NIHSS score was verified in our study to be related to the increase in 5-year mortality. Previous studies found that initial impaired consciousness was related to an increase in the mortality of AIS patients (10). As impaired 
Table 2 Outcomes of total AIS patients, and AIS patients with and without UGIB

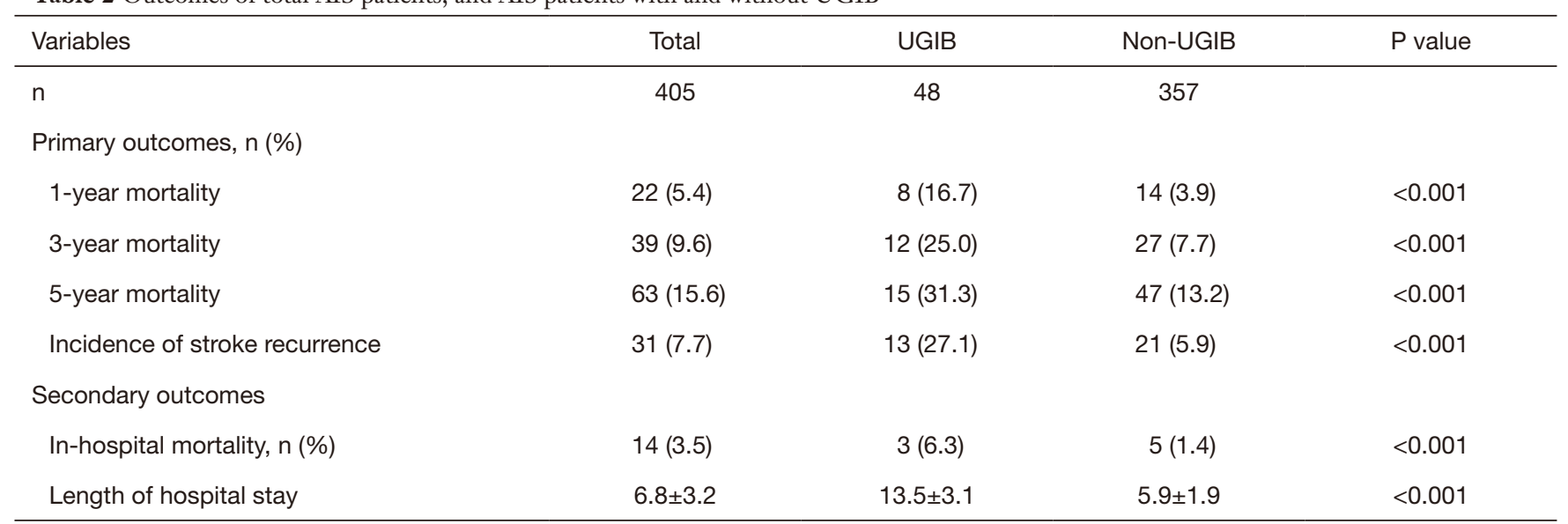

AIS, acute ischemic stroke; UGIB, upper gastrointestinal bleeding.

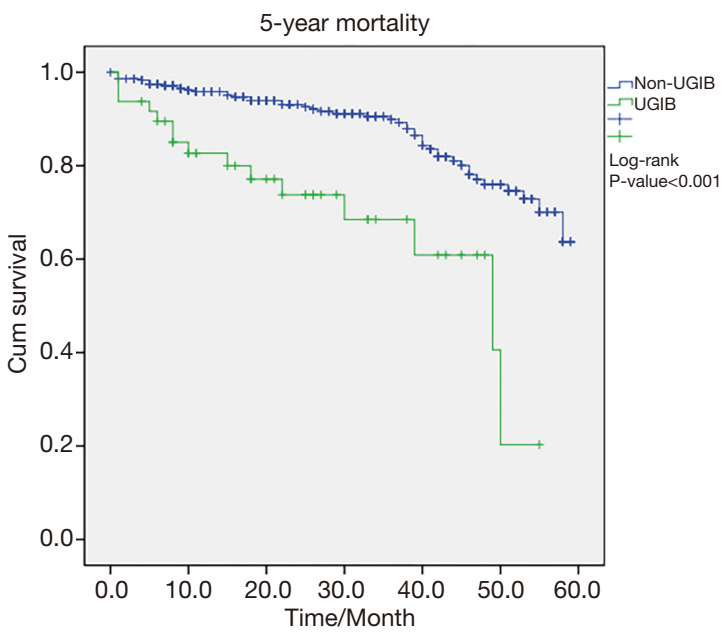

Figure 1 Kaplan-Meier curve of 5 -year mortality between AIS patients with and without UGIB. AIS, acute ischemic stroke; UGIB, upper gastrointestinal bleeding.

consciousness is only one item assessed by the NIHSS, we considered that the total NIHSS score was better than impaired consciousness alone in predicting the mortality of AIS patients. To some extent, stroke recurrence is also related to the severity of stroke. The recurrence of stroke was related to increased mortality in our study, and this was also found in previous studies $(20,21)$.

Because of the effects of stroke recurrence on the mortality of AIS patients, we found that risk factors of stroke recurrence were similar to those of 5-year mortality, such as UGIB, older age, and a high NIHSS score. Du et al. found many risk factors of stroke recurrence in

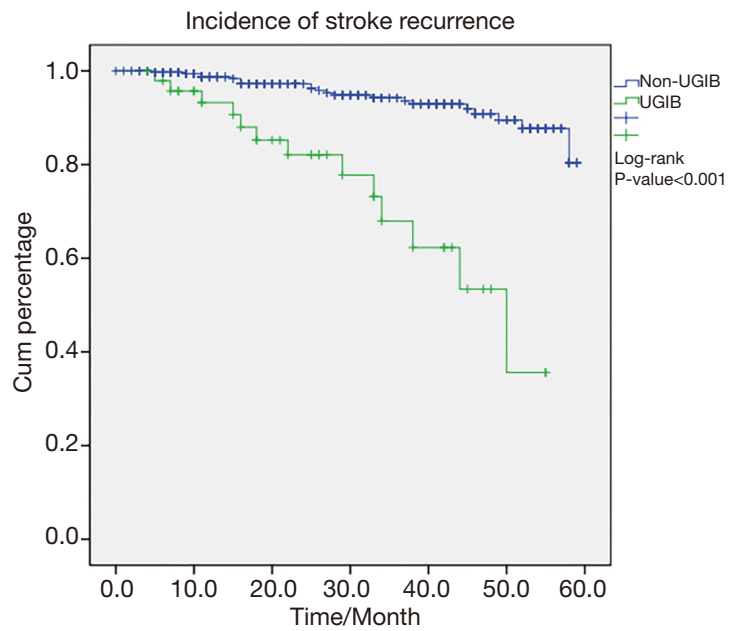

Figure 2 Kaplan-Meier curve of stroke recurrence incidence between AIS patients with and without UGIB. AIS, acute ischemic stroke; UGIB, upper gastrointestinal bleeding.

their study including age, gender, NIHSS score, diabetes mellitus, coronary heart disease, and atrial fibrillation (11). Hypertension was also verified as an independent risk factor of stroke recurrence in their study, which is similar to our findings. This can be explained by the fact that long term hypertension can lead to arteriosclerosis and increased fragility, thus making stroke recurrence more likely.

Considering the importance of UGIB in the prognosis of AIS patients, the specific predictors of UGIB need to be identified. We found that older age, a high NIHSS score, and previous anticoagulant use were predictors of UGIB in AIS patients. Previous studies have also identified these 
Table 3 Cox proportional hazards regression analysis of 5-year mortality in AIS patients

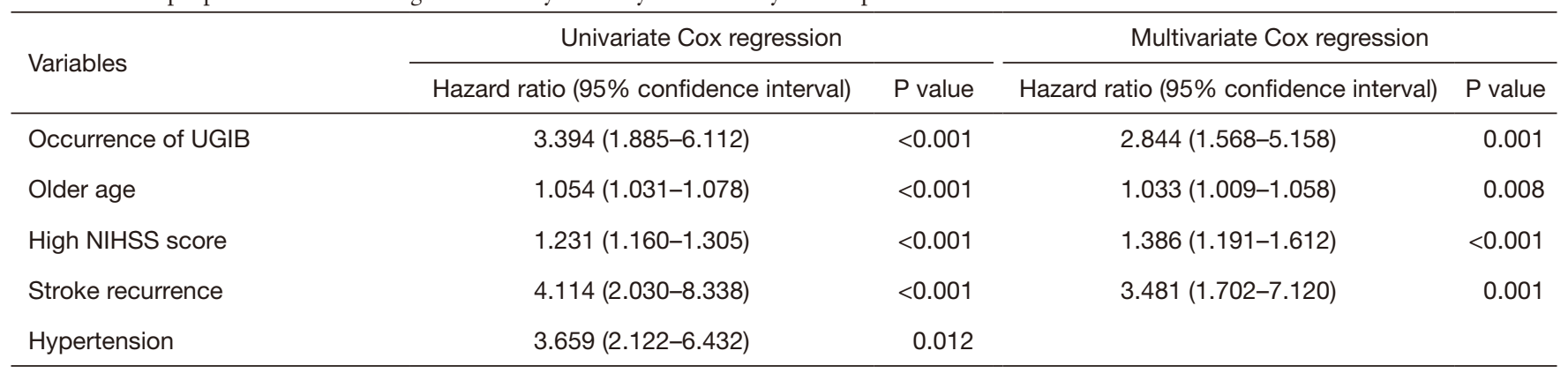

AIS, acute ischemic stroke; UGIB, upper gastrointestinal bleeding; NIHSS, National Institute of Health Stroke Scale.

Table 4 Cox proportional hazards regression analysis of recurrence in AIS patients

\begin{tabular}{|c|c|c|c|c|}
\hline Variables & \multicolumn{2}{|l|}{ Univariate Cox regression } & \multicolumn{2}{|l|}{ Multivariate Cox regression } \\
\hline Occurrence of UGIB & $6.336(3.125-12.845)$ & $<0.001$ & $2.929(1.354-6.337)$ & 0.006 \\
\hline Older age & $1.060(1.028-1.092)$ & $<0.001$ & $1.054(1.023-1.086)$ & 0.001 \\
\hline High NIHSS score & $1.262(1.177-1.354)$ & $<0.001$ & $1.221(1.014-1.469)$ & 0.035 \\
\hline Previous anticoagulant use & 3.189 (1.056-9.641) & 0.034 & & \\
\hline
\end{tabular}

AIS, acute ischemic stroke; UGIB, upper gastrointestinal bleeding; NIHSS, National Institute of Health Stroke Scale.

Table 5 Logistic regression analysis of predictors of UGIB in AIS patients

\begin{tabular}{lcccc}
\hline \multirow{2}{*}{ Variables } & \multicolumn{2}{c}{ Univariate Cox regression } & & Multivariate Cox regression \\
\cline { 2 - 3 } Gender & $1.842(0.955-3.553)$ & 0.068 & Hazard ratio (95\% confidence interval) & P value \\
Older age & $1.049(1.024-1.075)$ & $<0.001$ & $1.055(1.025-1.086)$ \\
High NIHSS score & $1.076(1.033-1.120)$ & $<0.001$ & $1.085(1.037-1.134)$ \\
PACI & $1.875(1.019-3.453)$ & 0.043 & $<0.001$ \\
TACl & $0.325(0.125-0.846)$ & 0.021 & $<0.001$ \\
Previous anticoagulant use & $3.019(1.405-6.487)$ & 0.005 & $2.556(1.135-5.754)$ \\
\hline
\end{tabular}

AIS, acute ischemic stroke; UGIB, upper gastrointestinal bleeding; NIHSS, National Institute of Health Stroke Scale; PACI, partial anterior circulation infarct; TACI, total anterior circulation infarct.

three variables as predictors of UGIB $(8,9,22)$. In addition, because hepatic cirrhosis is a coagulation disorder, it may also be related to the incidence of UGIB (8), but as only two patients in our study had cirrhosis this limited any further analysis. Stroke subtype may also affect the incidence of UGIB, however, the small population in our study limited the analysis of the relationship between stroke subtype and the incidence of UGIB.

There are some limitations in our study. We included 405 AIS patients, but only 48 patients had UGIB. Considering the low incidence of UGIB in AIS patients, a larger sample size may be beneficial to further analyze the effect of UGIB on prognosis in AIS patients. Also, as this was a retrospective study and all data were collected 
retrospectively, this may have led to some selection bias in our study which affected the results of the multivariate regression analysis. Moreover, we did not collect any data on the cause and treatment of UGIB, which may also be associated with the prognosis of AIS patients. Finally, detailed data concerning the dosage and duration of anticoagulant use, antiplatelet use, and NSAID use were not collected in this study, which may also be important factors affecting the incidence of UGIB.

\section{Conclusion}

Our study found that AIS patients with UGIB had a higher 5 -year mortality and a higher incidence of stroke recurrence than those without UGIB. Older age, a high NIHSS score, and previous anticoagulant use may be related to the incidence of UGIB in AIS patients. These results may provide some guidance for the treatment and nursing of AIS patients.

\section{Acknowledgments}

Funding: None.

\section{Footnote}

Reporting Checklist: The authors have completed the STROBE reporting checklist. Available at http://dx.doi. org/10.21037/apm-21-907

Data Sharing Statement: Available at http://dx.doi. org/10.21037/apm-21-907

Conflicts of Interest: All authors have completed the ICMJE uniform disclosure form (available at hhttp://dx.doi. org/10.21037/apm-21-907). The authors have no conflicts of interest to declare.

Ethical Statement: The authors are accountable for all aspects of the work in ensuring that questions related to the accuracy or integrity of any part of the work are appropriately investigated and resolved. All procedures performed in this study involving human participants were in accordance with the Declaration of Helsinki (as revised in 2013). The study was approved by institutional ethics board of Affiliated Hospital of Jiangnan University (No.: 02019050). Written informed consent from patients was not required due to the retrospective nature of the study.
Open Access Statement: This is an Open Access article distributed in accordance with the Creative Commons Attribution-NonCommercial-NoDerivs 4.0 International License (CC BY-NC-ND 4.0), which permits the noncommercial replication and distribution of the article with the strict proviso that no changes or edits are made and the original work is properly cited (including links to both the formal publication through the relevant DOI and the license). See: https://creativecommons.org/licenses/by-nc-nd/4.0/.

\section{References}

1. Ujeyl M, Koster I, Wille H, et al. Comparative risks of bleeding, ischemic stroke and mortality with direct oral anticoagulants versus phenprocoumon in patients with atrial fibrillation. Eur J Clin Pharmacol 2018;74:1317-25.

2. Stanton RJ, Eckman MH, Woo D, et al. Ischemic Stroke and Bleeding: Clinical Benefit of Anticoagulation in Atrial Fibrillation After Intracerebral Hemorrhage. Stroke 2020;51:808-14.

3. Proietti M, Lane DA, Boriani G, et al. Stroke Prevention, Evaluation of Bleeding Risk, and Anticoagulant Treatment Management in Atrial Fibrillation Contemporary International Guidelines. Can J Cardiol 2019;35:619-33.

4. Ogata T, Kamouchi M, Matsuo R, et al. Gastrointestinal bleeding in acute ischemic stroke: recent trends from the fukuoka stroke registry. Cerebrovasc Dis Extra 2014;4:156-64.

5. Mennuni MG, Halperin JL, Bansilal S, et al. Balancing the Risk of Bleeding and Stroke in Patients With Atrial Fibrillation After Percutaneous Coronary Intervention (from the AVIATOR Registry). Am J Cardiol 2015;116:37-42.

6. Lip GYH, Mauri L, Montalescot G, et al. Relationship of stroke and bleeding risk profiles to efficacy and safety of dabigatran dual therapy versus warfarin triple therapy in atrial fibrillation after percutaneous coronary intervention: An ancillary analysis from the RE-DUAL PCI trial. Am Heart J 2019;212:13-22.

7. Hamidon BB, Raymond AA. The risk factors of gastrointestinal bleeding in acute ischaemic stroke. Med J Malaysia 2006;61:288-91.

8. Ji R, Shen H, Pan Y, et al. Risk score to predict gastrointestinal bleeding after acute ischemic stroke. BMC Gastroenterol 2014;14:130.

9. Chen CM, Hsu HC, Chuang YW, et al. Study on factors affecting the occurrence of upper gastrointestinal bleeding in elderly acute stroke patients undergoing rehabilitation. J 
Nutr Health Aging 2011;15:632-6.

10. Chou YF, Weng WC, Huang WY. Association between gastrointestinal bleeding and 3-year mortality in patients with acute, first-ever ischemic stroke. J Clin Neurosci 2017;44:289-93.

11. Du W, Zhao X, Wang Y, et al. Gastrointestinal bleeding during acute ischaemic stroke hospitalisation increases the risk of stroke recurrence. Stroke Vasc Neurol 2020;5:116-20.

12. Berg DD, Ruff CT, Jarolim P, et al. Performance of the ABC Scores for Assessing the Risk of Stroke or Systemic Embolism and Bleeding in Patients With Atrial Fibrillation in ENGAGE AF-TIMI 48. Circulation 2019;139:760-71.

13. Ocak G, Noordzij M, Rookmaaker MB, et al. Mortality due to bleeding, myocardial infarction and stroke in dialysis patients. J Thromb Haemost 2018;16:1953-63.

14. He L, Zhang J, Zhang S. Risk factors of in-hospital mortality among patients with upper gastrointestinal bleeding and acute myocardial infarction. Saudi J Gastroenterol 2018;24:177-82.

15. Kyaw MH, Lau JYW. High Rate of Mortality More Than 30 Days After Upper Gastrointestinal Bleeding. Clin Gastroenterol Hepatol 2017;15:1858-9.

16. Vora P, Pietila A, Peltonen M, et al. Thirty-Year Incidence and Mortality Trends in Upper and Lower Gastrointestinal Bleeding in Finland. JAMA Netw Open 2020;3:e2020172.
17. Adoukonou T, Agbetou M, Bangbotche R, et al. LongTerm Mortality of Stroke Survivors in Parakou: 5-Year Follow-Up. J Stroke Cerebrovasc Dis 2020;29:104785.

18. Evans NR, Wall J, To B, et al. Clinical frailty independently predicts early mortality after ischaemic stroke. Age Ageing 2020;49:588-91.

19. Forti P, Maioli F, Nativio V, et al. Association of prestroke glycemic status with stroke mortality. BMJ Open Diabetes Res Care 2020;8:e000957.

20. Yasaka M, Koretsune Y, Yamashita T, et al. Recurrent Stroke and Bleeding Events after Acute Cardioembolic Stroke-Analysis Using Japanese Healthcare Database from Acute-Care Institutions. J Stroke Cerebrovasc Dis 2018;27:1012-24.

21. Antonenko K, Paciaroni M, Agnelli G, et al. Sex-related differences in risk factors, type of treatment received and outcomes in patients with atrial fibrillation and acute stroke: Results from the RAF-study (Early Recurrence and Cerebral Bleeding in Patients with Acute Ischemic Stroke and Atrial Fibrillation). Eur Stroke J 2017;2:46-53.

22. Rumalla K, Mittal MK. Gastrointestinal Bleeding in Acute Ischemic Stroke: A Population-Based Analysis of Hospitalizations in the United States. J Stroke Cerebrovasc Dis 2016;25:1728-35.

(English Language Editor: D. Fitzgerald)
Cite this article as: Wang T, Zhu D, Kong L, Mu C, Li C, $\mathrm{Hu}$ L. Effect of upper gastrointestinal bleeding on prognosis of middle-aged patients with acute ischemic stroke: a retrospective study. Ann Palliat Med 2021;10(5):5494-5501. doi: 10.21037/apm21-907 